Joseph A. Fisher MD FRCPC, Terry Martire RT, George A. Volgyesi PEng

\title{
Equipment
}

\section{Evaluation of a new pulse oximeter testing device}

\begin{abstract}
Background: Valid routine testing of pulse oximeters and their sensors is problematic. A suitable testing device must not only generate the pulsatile signal the pulse oximeter requires for its operation, but must possess light absorption characteristics similar to those of living tissue. A new device called Pulse Oximeter Tester (POT) has recently become available which, it is claimed, addresses these problems.

Purpose: To evaluate the POT as a suitable stimulus for pulse oximeters.
\end{abstract}

Method: We tested all the pulse oximeters and their sensors with a set of POTs simulating blood oxygen saturation of $80 \%$, $90 \%$ and $100 \%$. The tests were performed at simulated heart rates of 30,75 and $110 \mathrm{bpm}$.

Results: The $\mathrm{SpO}_{2}$ readings (mean $\pm \mathrm{SD}$ ) obtained with the $80 \%, 90 \%$ and $100 \%$ POTs were $80.7 \pm 1.3 \%, 90.3 \pm 0.9 \%$ and $100 \pm 0.0 \%$ respectively. There were no significant differences in readings obtained at the different simulated heart rates. Two pulse oximeters gave readings that deviated more than 2 SD from the mean. Their sensors were subsequently found to be defective.

Conclusion: POTs provide suitable stimuli for testing pulse oximeters. In our study sample they were found to be highly specific, but of unknown sensitivity.

Cadre: La vérification courante des oxymètres de pouls et de leur capteur suscite des problèmes. Un appareil adéquat pour leur vérification doit non seulement générer le signal pulsatile requis pour le fonctionnement de l'oxymètre mais doit aussi posséder des caractéristiques d'absorption identique à celles du tissu vivant. Un nouvel appareil commercialisé depuis peu,

\section{Key words}

MEASUREMENT TECHNIQUES: pulse oximetry, testing.

From the Department of Anaesthesia, Mount Sinai Hospital, 600 University Avenue, Toronto, Ontario, MSG 1 X5.

Address correspondence to: Dr. Joseph Fisher, Department of Anaesthesia, Mount Sinai Hospital, 600 University Avenue, Toronto, Ontario M5G 1 X5.

Phone: 416-586-5270. Fax: 416-586-8664.

Accepted for publication 25th September, 1995. le Pulse Oximeter Tester (POT) pourrait répondre à ces exigences.

Objectif: Evaluer le stimulus généré par le POT sur les oxymètres de pouls.

Méthode: Les auteurs ont vérifié les oxymètres et leur capteur avec des réglages de POT qui simulaient des saturation en oxygène de $80 \%, 90 \%$ et $100 \%$. Les épreuves ont été effectuées avec des fréquences cardiaques simulées de 30,75 et 110 bpm.

Résultats: Les lectures de la $\mathrm{SPO}_{2}$ (moyenne \pm ET) obtenues avec des réglages à $80 \%, 90 \%$ et $100 \%$ ont été respectivement de $80,7 \pm 1,3 \%, 90,3 \pm 0,9 \%$ et $100 \pm 0,0 \%$. Les lectures n'ont pas été modifiées par les fréquence cardiaques. Deux oxymètres de pouls ont donné des résultats dont l'E.T. était le double de la moyenne. Leur capteur a été trouvé défectueux par la suite.

Conclusion: Le POT fournit des stimuli adéquats pour la vérification des oxymètres de pouls. Notre étude les a trouvés hautement spécifiques mais leur sensibilité demeure inconnue.

Pulse oximeters in clinical use seldom undergo regular testing despite the fact that an undetected malfunction may lead to serious consequences. ${ }^{1.2}$ This is because there has been no satisfactory method of routinely testing pulse oximeters. As pulse oximeters rely on the presence of cardiac pulsations to function, the traditional approach to testing has been to compare pulse oximeter readings from living animals or humans ${ }^{3,4}$ with blood oxygen saturation obtained by direct arterial blood sampling. The saturation is lowered by having the subjects inspire hypoxic gas mixtures. This method is time consuming, inconvenient and expensive and under certain circumstances is unethical.

In addition to the problem of simulating a pulse, an in vitro test of pulse oximeters must deal with the issue of simulating tissue light absorption. Pulse oximeters transilluminate vascular tissue with light of two different wavelengths emanating from red and near infrared light emitting diodes (LEDs). The tissues absorb a constant portion of the light, while a variable portion is absorbed 


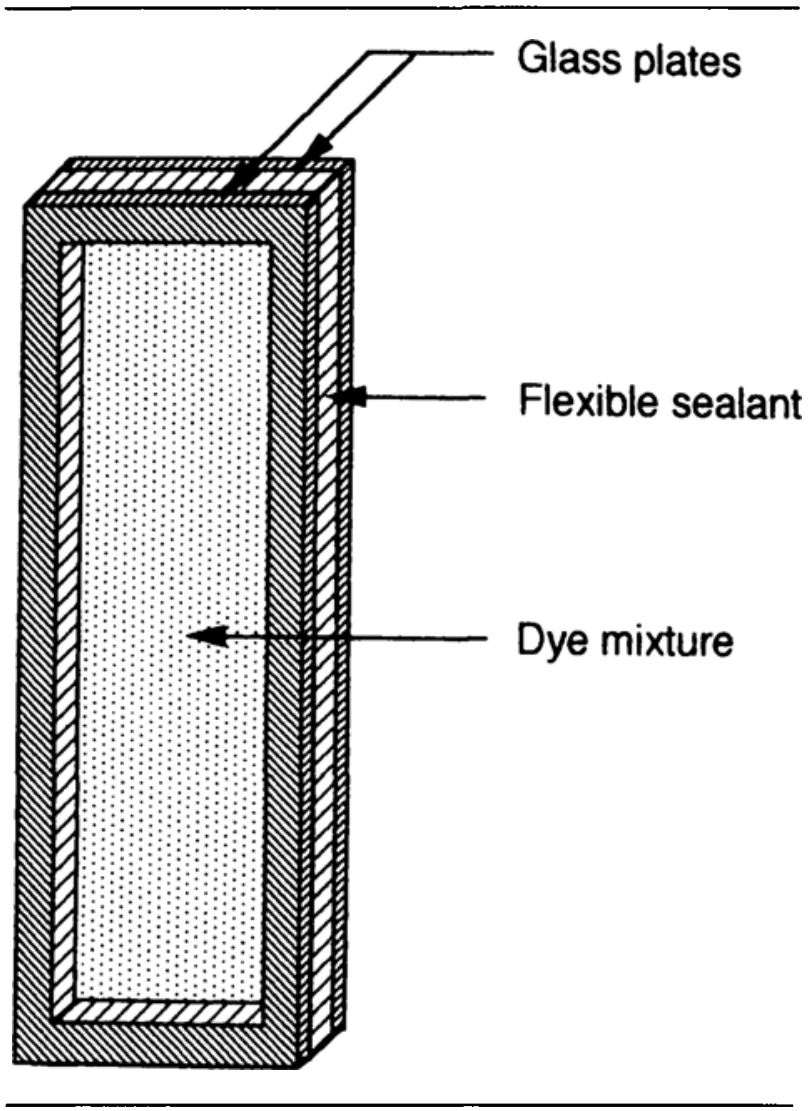

FIGURE 1 Construction of pulse oximeter tester. A liquid containing red and infrared absorbers is enclosed within two glass plates and sealed with a flexible sealant. Not shown is a plastic coating around the assembly.

by pulsating arterial blood. The intensity of the transmitted light is monitored by a sensor. The electrical signal representing absorption by arterial blood alone can be identified by subtracting the steady component of the signal due to tissue absorption from the combined signal. The saturation is then estimated by using an empirically derived relationship between the differential absorption of light at two specific wavelengths and blood oxygen saturation. The problem arises from the fact that the LEDs in sensors do not emit precise wavelengths, but may vary by $\pm 5 \mathrm{~nm}$. An in vitro testing method would, therefore, have to match the absorption patterns of blood at a given oxygen saturation for a wide spectrum of wavelengths of light.

Munley $e t$ al. ${ }^{5}$ described a plastic tube containing a single pigment that had a differential absorption of light at the red and near infrared wavelengths. It contained a rotating core to vary the amount of transmitted light and thus trigger the interpreting algorithm of the pulse oximeter. This generated reproducible readings for a given sensor, but the readings differed from sensor to sensor. Therefore, each sensor required its own custom built tester. Another approach was to use real blood circulated in a pulsatile fashion in complex circulatory system models. ${ }^{6.7}$ The use of blood in these methods makes them impractical as the long term stability of the blood cannot be maintained.

The difficulties in providing a precise mechanical stimulus for pulse oximeter sensors led several manufacturers to develop electronic devices which simulate the input from the sensor. This provides a test of the correct operation of the electronic circuitry only. A new microprocessor-based pulse oximeter tester (Bio-Tek Instruments, Inc. of Winoosky, Vermont) emits signals that simulate specific blood oxygen saturations to the pulse oximeter sensor. Although this system is an improvement over previous designs, it does not test for errors introduced by the wavelength variations in the light emitting diodes of individual pulse oximeter sensors.

A pulse oximeter tester (POT) has been recently described $^{8 . *}$ that contains a stable mixture of dyes approximating the light absorption characteristics of blood at three specific oxygen saturations. We evaluated the performance of this device as a uniform stimulus to pulse oximeters by applying it to a wide variety of combinations of pulse oximeters and sensors that may be found in clinical practice.

\section{Method}

The construction of each POT is detailed in Figure 1. The glass rectangles are attached along their perimeters by a flexible layer of black silicone rubber. The approximately $1 \mathrm{~mm}$ wide space between them is filled with a liquid containing a mixture of red and infrared absorbing substances. In order to protect the fragile glass assembly, a tough translucent rubber coating covers the POT. This coating also prevents the liquid from evaporating through the silicone seal, and reduces the effect of light reflection at the outer glass surfaces.

One of us (TM) tested all the pulse oximeters in clinical use at this hospital (Table) using the following procedure. Each POT was placed flat on a table top extending over the edge, allowing the sensor to be attached to the extended portion of the POT. Rhythmic pulsations were induced in the POT by pressing repeatedly with a finger on the portion resting on the table (Figure 2) until steady heart rate and $\mathrm{SpO}_{2}$ readings were obtained. Each pulse oximeter was tested at simulated heart rates of 30 , 75 and $110 \mathrm{bpm}$ with POTs designed to simulate blood oxygen saturations of 80,90 and $100 \%$. The $\mathrm{SpO}_{2}$ read-

*Prototype pulse oximeter tester developed. Biomedical Safety \& Standards, December 1, 1992. 
TABLE Variety and number of pulsc oximeters tested

\begin{tabular}{lc}
\hline Pulse oximeter & Number tested \\
\hline Criticare 504 & 1 \\
Datex 251 & 20 \\
Datex Trans. & 1 \\
H/P 78352C & 3 \\
Horizon 2000 & 4 \\
Miniox V & 1 \\
Nellcor N10 & 3 \\
Nellcor N20 & 1 \\
Nellcor N100 & 2 \\
Nellcor N200 & 29 \\
Nonin 8500 & 1 \\
Novametrix 505 & 2 \\
Protocol 102 EL & 1 \\
Siemens MicrO2 & 1 \\
Total & 70 \\
\hline
\end{tabular}

ings for each of the POTs at each of the three heart rates were recorded.

The light absorption spectra of the POTs used in the study were obtained using a Hitachi U-2000 spectrophotometer and compared with that of haemoglobin of corresponding oxygen saturation. The light absorption spectrum of haemoglobin at various oxygen saturations was estimated by linear interpolation of spectra of fully saturated and fully unsaturated haemoglobin. ${ }^{9}$

Statistical comparisons of saturation readings at each simulated heart rate were made using the ANOVA test. The criterion for significance was considered at $P<$ 0.05 .

\section{Results}

We tested 70 pulse oximeters. Readings were obtained with all pulse oximeters tested with each POT at each simulated heart rate. The mean $\mathrm{SpO}_{2}$ readings obtained with each POT using three different simulated heart rates were not statistically different (Figure 3 ). The frequency distribution of $\mathrm{SpO}_{2}$ readings at the simulated heart rate of 110 is illustrated in Figure 4. The mean and standard deviation of readings of $\mathrm{SpO}_{2}$ from the $80 \%$ and $90 \%$ POTs were $80.7 \pm 1.3$ and $90.3 \pm 0.9$ respectively.

Two pulse oximeters were found to give readings outside of two standard deviations from the mean for both the $80 \%$ and $90 \%$ POTs. To determine whether the fault in $2 / 70$ pulse oximeter-sensor combinations was with the POTs or the pulse oximeters, we tested the two pulse oximeters for proper function. The suspicion that these pulse oximeters were under-reading was confirmed by comparisons on several patients with properly functioning pulse oximeters. As well, replacement of the sensors

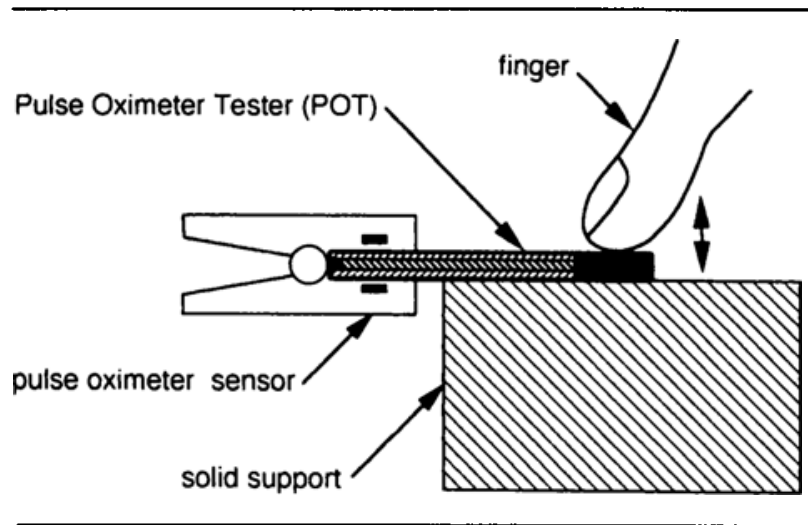

FIGURE 2 Method of testing pulse oximeters. Onc end of the POT rests on a solid support. The pulse oximeter sensor is placed on the other end. Pulsations are then simulated by pressing on the supported end of the POT.

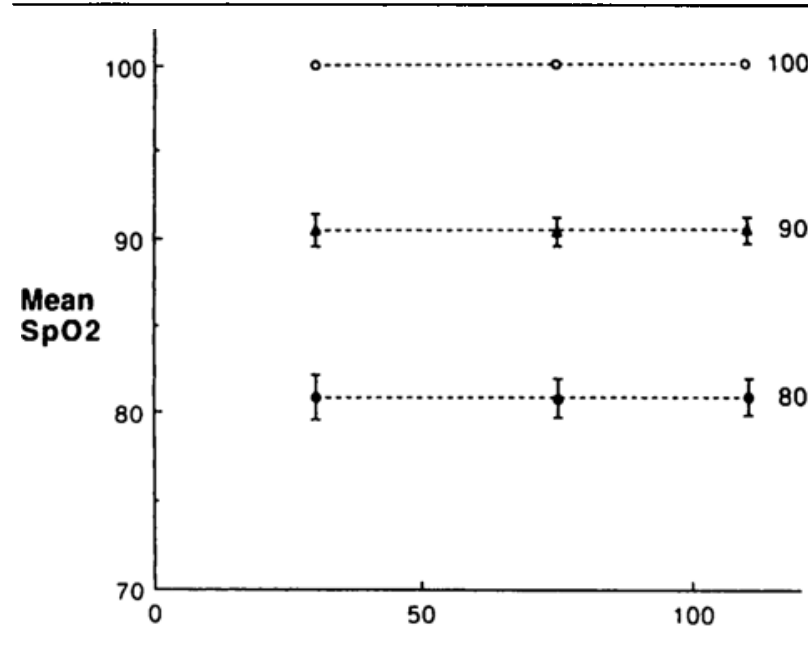

Simulated heart rate (BPM)

FIGURE 3 Mean and one standard deviation of pulse oximeter readings at simulated heart rates of 60,80 and $150 \mathrm{bpm}$. There was no difference among the $\mathrm{SpO}_{2}$ readings at the three simulated heart rates.

in each case resulted in readings with the POTs that were within two standard deviations of the mean.

The shape of the absorption spectra of the POTs simulating blood oxygen saturations of 80 and $90 \%$ were similar to those of haemoglobin of corresponding saturations in the 650 to $670 \mathrm{~nm}$ range (Figure 5).

\section{Discussion}

The POTs are promoted as providing uniform stimuli to pulse oximeter sensors. We used the 70 pulse oximeters in clinical use in our hospital to examine this claim. To test whether the POTs in fact provide a uniform stimulus to the probes, we assumed that the pulse oximeters 


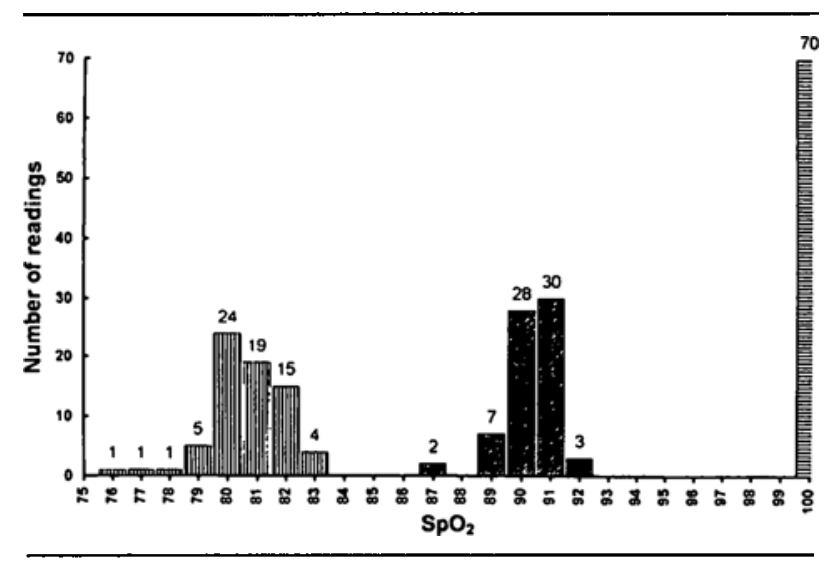

FIGURE 4 Frequency distribution of $\mathrm{SpO}_{2}$ readings obtained from 70 pulse oximeters with the three POTs at simulated heart rate of 110 . The readings of 76 and 77 with the 80 POT and the two readings of 87 with the 90 POT were <2 SD below their respective means and were obtained from the same two pulse oximeters. Vertical hatch: $80 \%$ POT; angled hatch: 90\% POT: horizontal hatch: 100\% POT.

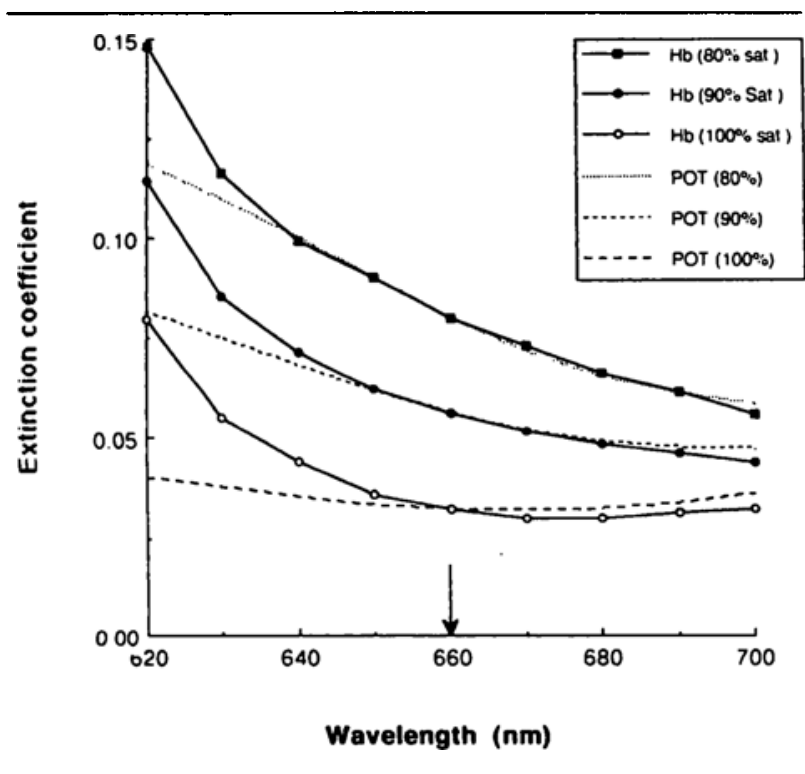

FIGURE 5 The shape of the absorplion spectra of the POTs are similar to those of hacmoglobin at oxygen saturations of $80 \%, 90 \%$ and $100 \%$ in the range of 650 to $670 \mathrm{~nm}$. To illustrate the similarity of absorption spectra of the dye mixtures and blood, the absorption spectrum of the dye mixture of each POT was matched to that of blood at the corresponding oxygen saturation at $660 \mathrm{~nm}$.

in clinical use were functioning properly. The clinical confirmation of a bias in readings of the two pulse oximeters that gave us the outlier values allows us to conclude that the stimulus is indeed uniform in our sample of pulse oximeters.

The wavelength of the red LED contained in each pulse oximeter sensor may deviate by $\pm 5 \mathrm{~nm}$ from the specified value of $660 \mathrm{~nm}$. The degree of light absorption by haemoglobin at a given saturation differs within a small range around this wavelength. To compensate for this, manufacturers of pulse oximeters either use only LEDs whose wavelengths are very close to the specified value, or code the sensor with the actual wavelength of the red LED and then electronically correct for the deviation from the specified value. A previous study using POTs whose dye absorption spectra were not as carefully matched to those of haemoglobin reported a larger variability of $\mathrm{SpO}_{2}$ measured with a variety of sensors. ${ }^{8}$ Although we did not measure the wavelength of the light emitted by the sensors, it is assumed that the usual large variation in outputs of these devices was represented in our sample, yet we found a very small variation of $\mathrm{SpO}_{2}$ readings. The variation in light absorption by haemoglobin at a given saturation, near the wavelength of the near infrared LED (nominally set at 940 $\mathrm{nm}$ ) is small. For this reason, the variation of wavelength emitted by this LED has little effect on $\mathrm{SpO}_{2}$. The uniformity of readings obtained with the $80 \%$ and 90\% POTs indicates that the absorption spectra of the dyes for the range of wavelengths emitted by the sensors were similar to that of haemoglobin at the corresponding oxygen saturations.

The $100 \%$ POT is designed to provide a pattern of absorption that is interpreted by the pulse oximeter algorithm to be slightly above $100 \%$. The manufacturer states that this POT is provided only to confirm that the pulse oximeter can display a readout of $100 \%$. The ability of a pulse oximeter to discriminate in the 95 to $100 \%$ saturation range may be important in paediatric anaesthesia, where retrolental fibroplasia is a consideration.

As both abnormal ( $>2$ SD below the mean) readings came from pulse oximeters that were shown to be faulty, suggests that there is a high specificity to the POT as a test of pulse oximeters. Without an independent test for proper.functioning of pulse oximeters that gave readings within 2 SD of the designated POT, we are unable to comment on the sensitivity of the POTs in identifying malfunctioning pulse oximeters. It is, however, reasonable to assume that these pulse oximeters were functioning properly as they were in constant clinical use and gave results consistent with the clinical situation.

\section{Conclusion}

The new pulse oximeter tester examined in this study is a commercially available in vitro device designed to test both the pulse oximeter and its sensor. We found that it provides a set of uniform stimuli simulating haemoglobin oxygen saturations of $80 \%, 90 \%$ and $100 \%$ to a wide variety of pulse oximeter sensors despite the 
assumed variability in the sensors' output wavelength. As a test of pulse oximeters, we found it highly specific but of unknown sensitivity. Considering the ease and use of this device, we feel that it may be useful as a practical routine tester for all pulse oximeters.

\section{Addendum}

Since this study was completed, Nonin Medical Inc. of Plymouth, MN has acquired exclusive worldwide rights to the POT, and is marketing an improved pulse oximeter testing system under the trade name of "Finger Phantom."

\section{References}

1 Broome $1 J$. A dangerous failure of a pulse oximeter. Anaesthesia 1990; 45: 166.

2 Spiers $S P W$, Harris $R W$. Failure of an Ohmeda Oxicap 4700. Anaesthesia 1991; 46: 420.

3 Russell GB, Graybeal JM. Accuracy of laminated disposable pulse-oximeter sensors. Respir Care 1995; 40: 728-33.

4 Severinghaus $J W$, Naifeh $K H$. Accuracy of response of six pulse oximeters to profound hypoxia. Anesthesiology 1987; 67: 551-8.

5 Munley AJ, Sik MJ, Shaw A. A test object for assessing pulse oximeters. Lancet 1989; 1: 1048-9.

6 Mendelson Y, Kent JC. An in vitro tissue model for evaluating the effect of carboxyhemoglobin concentration on pulse oximetry. IEEE Transactions on Biomedical Engineering 1989; 36: 625-7.

7 Reynolds KJ, Moyle JTB, Sykes MK, Hahn CEW. Response of 10 pulse oximeters to an in vitro test system. Br J Anaesth 1992; 68: 365-9.

8 Volgyesi GA, Suzuki Y, Lerman J. A simple testing device for pulse oximeters. Anesthesiology 1992; 77: A578.

9 de Kock JP, Reynolds KJ, Tarassenko L, Moyle JTB. The effect of varying LED intensity on pulse oximeter accuracy. Journal of Medical Engineering and Technology 1991; 15: $111-6$ 